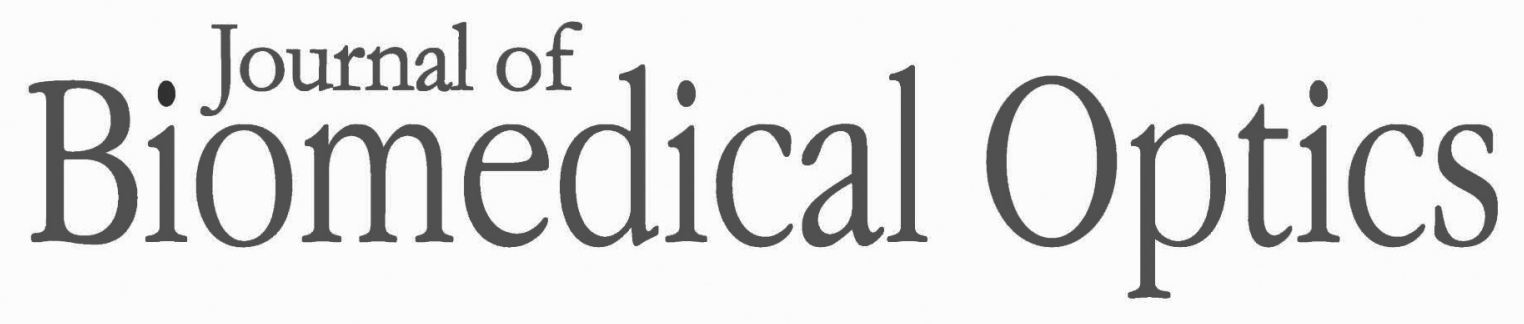

SPIEDigitalLibrary.org/jbo

\title{
Optodynamic energy-conversion efficiency during an Er:YAG-laser-pulse delivery into a liquid through different fiber-tip geometries
}

Peter Gregorčič Matija Jezeršek Janez Možina 


\title{
Optodynamic energy-conversion efficiency during an Er:YAG-laser-pulse delivery into a liquid through different fiber-tip geometries
}

Peter Gregorčič, Matija Jezeršek, and Janez Možina

University of Ljubljana, Faculty of Mechanical Engineering, Aškerčeva 6, SI-1000 Ljubljana, Slovenia

\begin{abstract}
When an erbium-laser pulse is directed into water through a small-diameter fiber tip (FT), the absorption of the laser energy superheats the water and its boiling induces a vapor bubble. We present the influence of different FT geometries and pulse parameters on the vapor-bubble dynamics. In our investigation, we use a free-running erbium: yttrium aluminum garnet (Er:YAG) $(\lambda=2.94 \mu \mathrm{m})$ laser that was designed for laser dentistry. Its pulse is directed into the water through FTs with a flat and conical geometry. Our results show that in the case of the conical FT, a spherical bubble is induced, while a channel-like bubble develops for the flat FT. The ratio between the mechanical energy of the liquid medium and the pulse energy, which we call the optodynamic energy-conversion efficiency, is examined using shadow photography. The results indicate that this efficiency is significantly larger when a conical FT is used and it increases with increasing pulse energy and decreasing pulse duration. The spherical bubbles are compared with the Rayleigh model in order to present the influence of the pulse duration on the dynamics of the bubble's expansion. @ 2012 Society of Photo-Optical Instrumentation Engineers (SPIE). [DOI: 10.1117/1.JBO.17.7.075006]
\end{abstract}

Keywords: erbium-laser pulse; cavitation bubble; vapor-bubble formation; shadowgraphy; fiber-tip geometry; laser-tissue interaction; lasers in medicine; optodynamics.

Paper 12108 received Feb. 13, 2012; revised manuscript received May 29, 2012; accepted for publication Jun. 1, 2012; published online Jul. 9, 2012.

\section{Introduction}

Lasers with wavelengths in the interval 2 to $3 \mu \mathrm{m}$ are widely used in various medical applications where clinical treatments are usually performed in a liquid environment. ${ }^{1-5}$ Of all the infrared lasers, the erbium: yttrium aluminum garnet (Er:YAG) laser, emitting pulsed light at $2.94 \mu \mathrm{m}$, has the highest absorption in water. ${ }^{6}$ Thus, its radiation delivered through a smalldiameter fiber tip (FT) can be used for a wide range of laser-assisted medical applications, such as lithotripsy, ${ }^{7}$ ophthalmology, ${ }^{8,9}$ and dentistry. ${ }^{10-17}$

Due to the strong absorption of erbium radiation in water, a vapor bubble is formed during the erbium-pulse delivery through an optical fiber that is not in contact with any tissue. ${ }^{18,19}$ The cavitation-bubble dynamics then affects the tissue-ablation efficiency in two ways. ${ }^{20}$ First, the bubble creates a vapor-water channel for the laser radiation from the FT to the tissue. ${ }^{21,22} \mathrm{Sec}$ ond, the bubble dynamics ${ }^{23-27}$ may contribute to the material's removal and collateral effects. ${ }^{28,29}$ The described phenomena represent a typical optodynamic process, where the energy of the laser pulse is coupled with the mechanical energy of the liquid medium. ${ }^{30}$ The characterization of these phenomena is therefore important for the optimization of biomedical processes based on laser ablation in liquids.

The main goal of our investigation is to study the influence of the FT geometry and the erbium-laser-beam parameters on the optodynamic (OD) energy-conversion efficiency, which we define as the mechanical energy of optically induced motion divided by the pulse energy. Here, the radiation of the Er:YAG

Address all correspondence to: Peter Gregorčič, University of Ljubljana, Faculty of Mechanical Engineering, Aškerčeva 6, SI-1000 Ljubljana, Slovenia. Tel: +38614771172; E-mail: peter.gregorcic@fs.uni-lj.si laser is delivered into an infinite liquid through FTs with a flat and conical geometry. In many biomedical applications, ${ }^{2,7,10,18,22,31}$ the flat FTs are applied. In this case, the laser radiation exits the optical fiber in forward direction with a relatively small divergence. On the other hand, in the past few years, the conical FTs are used in dentistry for cleaning, shaping, and sterilization of root canals. ${ }^{14-17}$ In this case, the conical FT is placed into the coronal portion of the pulpa chamber, filled with a liquid, such as water or sodium hypochlorite. ${ }^{12,17}$ For these dental treatments, there is a need to maximize the mechanical effects with minimal thermal side effects. ${ }^{16}$

Using shadow photography, we show that the shape of the bubble and its dynamics depend on the FT geometry. In the case of the flat FT, a channel-like bubble is induced, ${ }^{3,9,21,22,32}$ while our measurements show that a spherical bubble is developed when erbium radiation is delivered through the conical FT. Thus, we compare the measurements obtained using the conical FT with the Rayleigh model ${ }^{33}$ in dimensionless form to analyze the influence of the pulse duration on the bubble's dynamics.

Our measurements indicate that the stress waves are not induced during the bubble's formation. Therefore, in the case of the free-running mode Er:YAG pulses, the total mechanical energy of the liquid equals the energy of the first bubble's expansion. With these measurements, we can explain how the FT's geometry, the laser-pulse energy, and the pulse duration influence the cavitation bubble size, which further determines the acoustic transient amplitudes. ${ }^{32,34-37}$ The experiments were performed with a commercially available laser for clinical applications in laser dentistry. Thus, the experimental parameters are very similar to the parameters in clinical practice. 


\section{Materials and Methods}

\subsection{Experimental Setup}

Our experimental setup is schematically shown in Fig. 1. A free-running Er:YAG laser, designed for laser dentistry $(\lambda=2.940 \mu \mathrm{m}$, Fotona d.d., Slovenia, LightWalker), was used as the excitation source. We used pulses with a duration in the range $t_{L}=40-160 \mu \mathrm{s}$ and pulse energies (at the output of a FT) between $E_{L}=5-100 \mathrm{~mJ}$. The repetition was set to $5 \mathrm{~Hz}$. The excitation-pulse power as a function of time is detected by a $60-\mathrm{MHz}$ InAs-photodiode (PD-InAs) placed behind the rear mirror of the laser resonator (LR). The delivery of the laser pulse into the vessel with distilled water is performed through an articulated arm. Within the handpiece, the pulse is optically coupled into an interchangeable FT. The FT's end is placed $h=10 \mathrm{~mm}$ below the water level, as is schematically shown in the inset of Fig. 1. In our experiments, we used FTs with flat and conical terminations. Their geometries are schematically presented in Fig. 2. The FTs are made from fused silica with a low $\mathrm{OH}$-content core, a fluorine silica cladding and a polyimide jacket. Their numerical aperture in air is $\mathrm{NA}=0.22$. Thus, the light exits the flat FT conically with a maximum angle of $\psi_{\text {out }}=12.7 \mathrm{deg}$. Using the core refractive index $n_{c}=1.421$, the maximum inner angle is calculated to

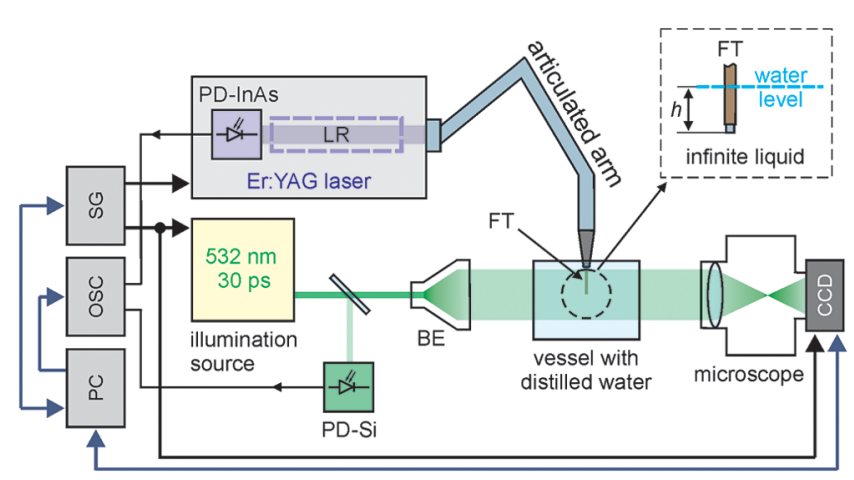

Fig. 1 Schematic view of the experimental setup.

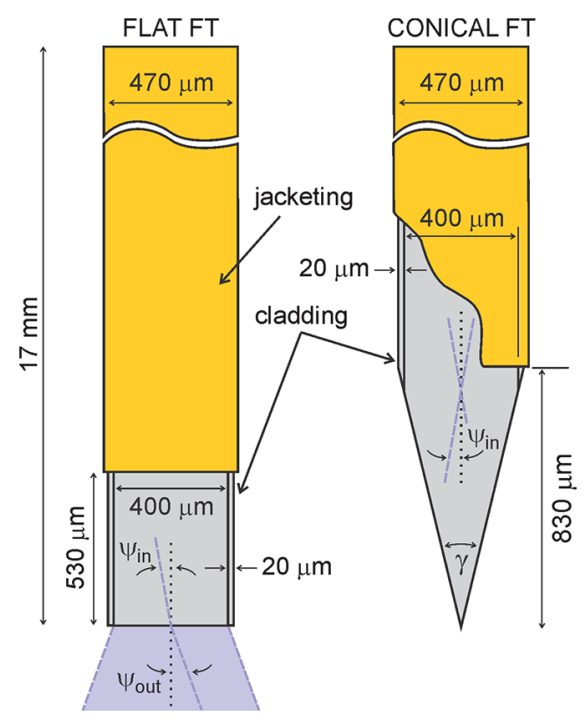

Fig. 2 The geometry of the fiber tips. be $\psi_{\text {in }}=8.9 \mathrm{deg}$. The conical FT's termination subtends an angle at $\gamma=27 \mathrm{deg}$.

The optodynamic phenomena in a liquid ${ }^{24,30,38,39}$ induced by erbium radiation delivered through different FTs were examined using shadowgraphy. Here, a frequency-doubled Nd:YAG laser (Ekspla, Lithuania, PL2250-SH-TH) emitting green $(\lambda=532 \mathrm{~nm})$ pulses with a duration of $30 \mathrm{ps}$ was used as the illumination source. The probe is partially reflected by a dielectric plate to the $1-\mathrm{GHz}$ Si-photodiode (PD-Si). A beam expander (BE) is placed in front of the illumination area where the laser-pulse-water interaction takes place. The image is captured through a microscope equipped with a charge-coupled device (CCD) camera (Basler AG, Germany, scA1400-17fm, $1.4 \mathrm{Mpx}$ ). The spatial resolution of our optical system was in the range of $\kappa=3.6-5.7 \mu \mathrm{m} /$ pixel. For a particular series of measurements, the $\kappa$ value was determined using the images of a calibrating pattern.

The lasers and the camera were synchronized with a signal generator (SG; Tektronix, US, AFG 3102) connected to a personal computer (PC). The experimental setup is automatically controlled with custom-developed software that also enables the setting of the excitation-laser parameters, the data acquisition from a digital oscilloscope (OSC; LeCroy, US, $600 \mathrm{MHz}$ Wave Runner 64MXi-A), image acquisition as well as data and image processing.

\subsection{Data Analysis}

Using shadowgraphy, we measured the vapor-bubble dynamics for various parameters $E_{L}$ and $t_{L}$. A sequence of images from multiple events was recorded by varying the time delay between the excitation pulse and the probe pulse. This delay is set by the SG and measured from the signals of the photodiodes PD-InAs and PD-Si.

The bubble locally changes the refractive index of the liquid. Consequently, a refractive-index gradient appears on its wall, representing the boundary between the steam and the surrounding water. ${ }^{38}$ This results in the deflection of the probe pulse casting a shadow, ${ }^{40}$ where the FT and cavitation bubble are visible as a black area on a bright background (e.g., see Fig. 3). The volume of the bubble during its expansion and collapse is estimated using the image processing of the shadowgraphs captured by the CCD. Here, we assume that the cavitation bubble is cylindrically symmetrical around the FT axis.

First, the grayscale (8-bit) image is converted into a blackand-white (1-bit) image. In each row, $l$ of the 1-bit image the edges are detected using horizontal gradients. Then, the radius $r_{l}$ is calculated as:

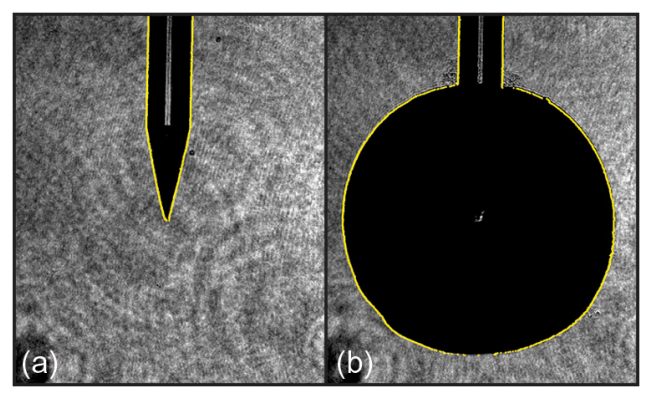

Fig. 3 Software detection of vertical edges. The yellow points show the very left and the very right edges of an individual line of the image. (a) The FT before the bubble formation. (b) A fiber tip with a cavitation bubble reaching its maximum volume. 


$$
r_{l}=\kappa \frac{x_{R l}-x_{L l}}{2}
$$

where, $x_{R l, L l}$, are the $x$ positions of the very left and the very right edge (the yellow points in Fig. 3), separately. From the radii, $r l$, the volume from the selected area is obtained by summing the one-pixel-wide slices:

$$
V=\kappa \pi \sum_{l=1}^{N} r_{l}^{2},
$$

where, $N$ is the height of the image in pixels. Finally, the bubble's volume $V_{B}$ is calculated as:

$$
V_{B}=V-V_{F T}
$$

Here, the volume of the FT, $V_{\mathrm{FT}}$, is determined from the images before the bubble's formation [e.g., see Fig. 3(a)] using Eqs. (1) and (2).

The pulse energy $E_{L}$ and its duration $t_{L}$ are simultaneously measured during the experiments using the signal $U(t)$ from the photodiode PD-InAs. Assuming a linear response of the photodiode, the pulse power $P(t)$ is proportional to the measured voltage $U(t)$ :

$$
P(t)=C U(t)
$$

and the pulse energy as a function of time is obtained as:

$$
E_{L}(t)=C \int_{0}^{t} U\left(t^{\prime}\right) \mathrm{d} t^{\prime}
$$

The proportionality constant $C$ is previously determined by employing a calibrated energy meter (Ophir Optronics Inc., Israel, Smart head to USB interface with a pyroelectric sensor PE50BF-DIF-SH-U2) at the output of the FT in air.

Figure 4 shows two typical graphs of power as a function of time for excitation pulses of the same energy $E_{L}=20 \mathrm{~mJ}$, but different pulse durations: $t_{L}=60 \mu \mathrm{s}$ (the solid curve) and $t_{L}=$ $120 \mu \mathrm{s}$ (the broken curve). The curves show an average of 50 measurements and the shaded areas are within one standard deviation from the mean.

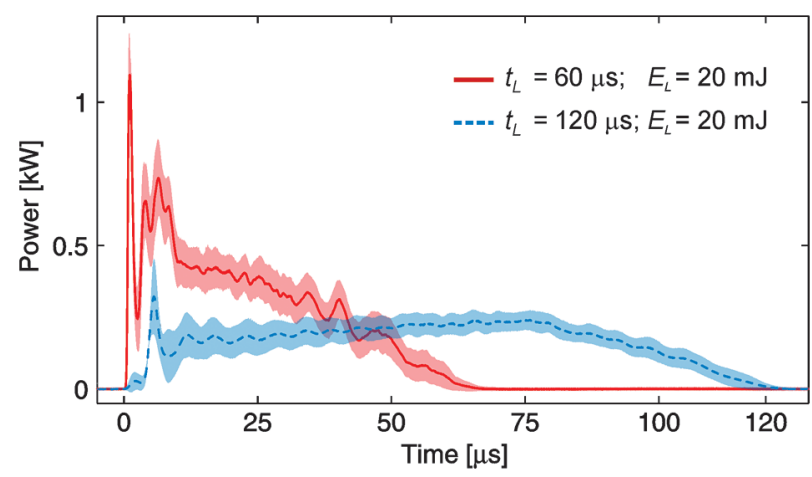

Fig. 4 Pulse power (an average of 50 measurements) as a function of time for laser energy $E_{L}=20 \mathrm{~mJ}$, and pulse durations $t_{L}=60 \mu \mathrm{s}$ (the solid curve) and $t_{L}=120 \mu \mathrm{s}$ (the broken curve), respectively. The shaded region shows a standard deviation.

\section{Results and Discussion}

\subsection{Bubble Formation}

The erbium radiation $(\lambda=2.94 \mu \mathrm{m})$ delivered into liquid water through a submerged FT is completely absorbed right beside the FT due to the high absorption coefficient $\mu_{a}=1.247 \times$ $10^{6} \mathrm{~m}^{-1}$. Thus, the water is instantly heated over the boiling point and a vapor bubble starts to form at the FT [e.g., see the second images in Fig. 5(a) and 5(b)] within the time interval $t_{v f}$ after the excitation-pulse initiation.

Figure 5 shows typical images of the vapor-bubble formation during the first $6 \mu \mathrm{s}$ after the beginning of the excitation pulse $\left(E_{L}=20 \mathrm{~mJ}, t_{L}=100 \mu \mathrm{s}\right)$. The time of the vapor formation $\left(t_{v f}\right)$ depends on the pulse energy and the pulse duration. However, in the case of the laser parameters used in our experiments, we found from the shadowgraphs that the vapor formation starts within the time interval $t_{v f}<1.5 \mu$ s, i.e., during the first spike of the laser. This observation is consistent with the experimental results presented by Brinkmann et al. ${ }^{1}$

\subsubsection{Flat fiber tip}

When a FT with the flat end [Fig. 5(a)] is used, the light exits the fiber within the cone defined by the angle $\psi_{\text {out }}$ (see Fig. 2). Owing to the strong absorption of the erbium radiation in water, the heated layer of a liquid has a thickness of $1 / \mu_{a}$, and the initially heated volume can be approximated by $V_{v f}=\frac{\pi R_{c}^{2}}{\mu_{a}}$, where, $R_{c}$ stands for the radius of the FT core. The characteristic thermal diffusion time, $t_{d}$, is defined as: ${ }^{41}$

$$
t_{d}=\frac{1}{D \mu_{a}^{2}} \approx 5 \mu \mathrm{s},
$$

where $D=1.4 \times 10^{-7} \mathrm{~m}^{2} \mathrm{~s}^{-1}$ stands for the thermal diffusivity of water. In our case $t_{v f}<t_{d}$, so the vapor is induced under adiabatic conditions. Therefore, the average local temperature within the heated volume $V_{v f}$ before the vapor formation can be estimated as:

(a)

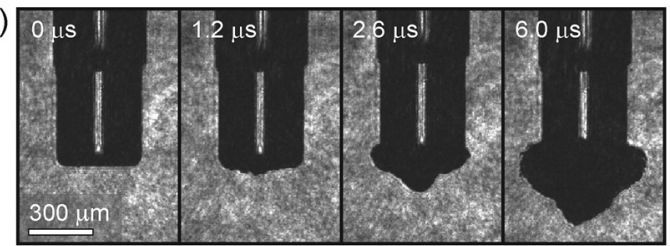

(b)

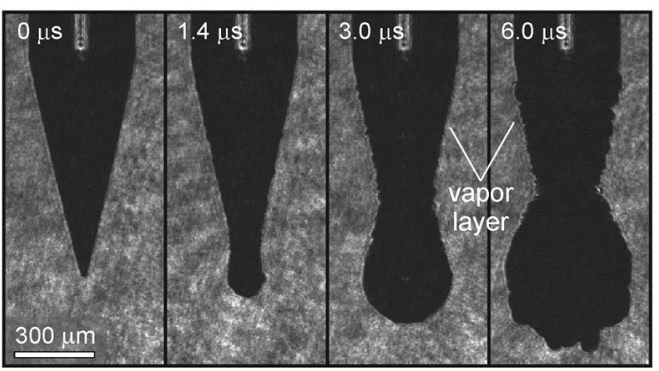

Fig. 5 Typical images of vapor-bubble formation in water in the first $6 \mu \mathrm{s}$ after the beginning of the excitation-pulse radiation $\left(E_{L}=20 \mathrm{~m}\right.$, $t_{L}=100 \mu \mathrm{s}$ ) delivered through the (a) flat and (b) conically terminated FT. The scale is shown by a bar on the first image. 


$$
T_{v f}=T_{0}+0.63 \frac{E_{L}\left(t_{v f}\right) \mu_{a}}{\pi R_{c}^{2} \rho c_{v}} .
$$

In the above equation, $T_{0}=298 \mathrm{~K}$ stands for the initial temperature of the water, $\rho$ is the density of the water, $c_{v}$ is the specific heat capacity at a constant volume, $E_{L}\left(t_{v f}\right)$ is the irradiated laser energy before the vapor formation, and the factor 0.63 is the portion of energy $E_{L}\left(t_{v f}\right)$ absorbed in the layer with the thickness $1 / \mu_{a}$.

Figure 6(a) shows a typical temporal power-evolution of the excitation pulse (after the FT) for the case in Fig. 5. The energy as a function of time for the first $14 \mu \mathrm{s}$ [the shaded area in Fig. 6(a)] is calculated using Eq. (5) and shown in Fig. 6(b). The red point at $t_{v f}=1.2 \mu$ s in Fig. 6(b) corresponds to the time of the boiling point determined from the second image in Fig. 5(a), where the first signs of the bubble formation are visible. Here, a series of images captured in the first $2 \mu$ s and separated by $0.2 \mu$ s were analyzed. However, an on-line determination of the boiling point can be performed using an optical monitoring proposed by Brinkmann et al. ${ }^{1}$

Using Eq. (7) and the irradiated laser energy $E_{L}\left(t_{v f}\right)=$ $0.14 \mathrm{~mJ}$ [the red point in Fig. 6(b)], we estimate for the flat FT that the average water temperature within the volume $V_{v f}$ before vaporization is around $230^{\circ} \mathrm{C}$. Thus, the bubble is formed by the explosive boiling ${ }^{20}$ of a superheated water layer.

\subsubsection{Conical fiber tip}

The situation is more complex when a conical FT is used. Because of the conical geometry, the light experiences several reflections and is gathered at the bottom of the FT's conical end, as explained below.

Here, the light enters the conical termination within the cone defined by the maximum inner angle $\psi_{\text {in }}$ (see Fig. 2). This angle is the same as in the case of the flat geometry. Then it reaches the
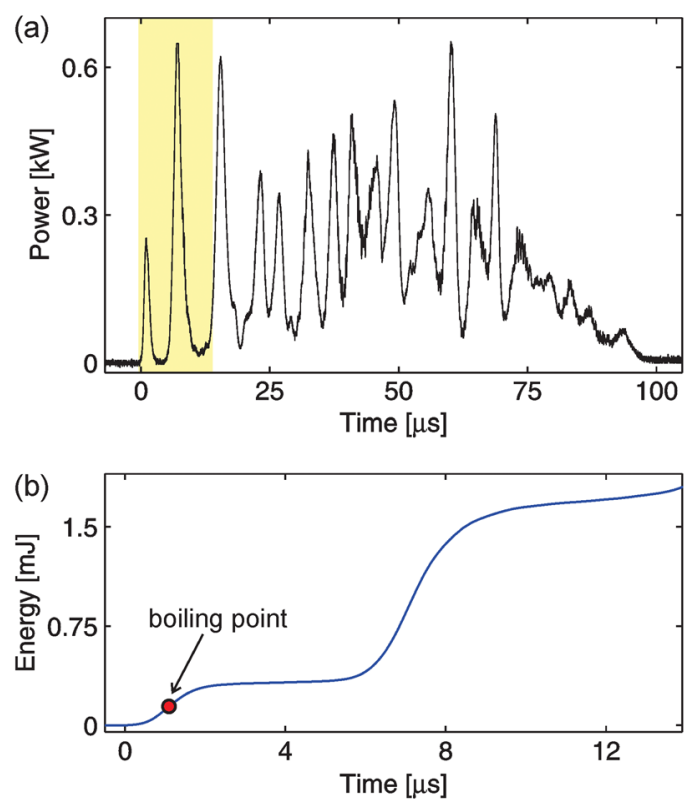

Fig. 6 (a) Pulse power as a function of time for the laser energy $E_{L}=20 \mathrm{~mJ}$ and pulse duration $t_{L}=100 \mu \mathrm{s}$. (b) Pulse energy as a function of time for the first $14 \mu \mathrm{s}$ [the shaded area in (a)]. The red point shows the time when a layer of vapor in the second image of Fig. 5(a) is induced. conical-end boundary making an angle $\alpha_{0}=90^{\circ}-\frac{\gamma}{2}-\delta$ with the boundary's normal (see Fig. 7). The angle between the entering light and the FT axis, $\delta$, is in the range $\delta \in\left[-\psi_{\mathrm{in}},+\psi_{\mathrm{in}}\right]$.

Depending on the refractive indices of the fiber core $\left(n_{c}\right)$ and the surrounding medium $\left(n_{m}\right)$, and the angles $\gamma$ and $\delta$, the light is totally or partially reflected. The critical angle for total internal reflection $\alpha_{\text {tot }}$ is defined as $\arcsin \left(n_{m} / n_{c}\right)$. All the rays that are incident at angles greater than $\alpha_{\text {tot }}$ are totally reflected and bounded within the FT. However, after the first incidence at an angle $\alpha_{0}$ with respect to the boundary's normal, the reflected light is incident upon the conical-end boundary again, but with the smaller angle $\alpha_{1}$. This repeats several times and the angle of incidence $\alpha_{m}$ after the $m$-th reflection decreases as:

$$
\alpha_{m}=\alpha_{0}-m \gamma ; \quad m_{\max }=\left\lfloor 2 \alpha_{0} / \gamma\right\rfloor .
$$

After the $m_{\max }$-th reflection, the reflected light returns into the FT (see Fig. 7). During each reflection, the portion of reflected light is defined by the Fresnel laws. ${ }^{9}$

In our case, the first incidence angle $\alpha_{0}$ exceeds $67.6 \mathrm{deg}$, while the angle of the total internal reflection for the silicawater interface equals $\alpha_{\mathrm{totSW}}=63.7 \mathrm{deg}$, using the refractive index of water $n_{w}=1.274$ at $\lambda=2.94 \mu \mathrm{m} .{ }^{6}$ Thus, the angle of the first incidence $\alpha_{0}$ is larger than $\alpha_{\text {totsw }}$, so the whole light is first totally reflected from the conical-end boundary. For this reason, the light gathers at the bottom part of the conical FT and exits the FT at different angles defined by Snell's law. ${ }^{9,42}$ Therefore, the power density at the exit of the conical FT is increased in comparison with the flat FT.

Although the condition for total internal reflection is fulfilled for the first incidence of laser radiation upon the silica-water boundary, a vapor layer is observed at $t>3 \mu \mathrm{s}$ along the whole conical-termination [see Fig. 5(b)]. We believe that this happens because the conical termination of the FT is not perfectly polished, and due to the evanescent field. Therefore, a portion of the light exits the FT also during the first incidence and turns a thin layer of water into vapor. When the conical-end

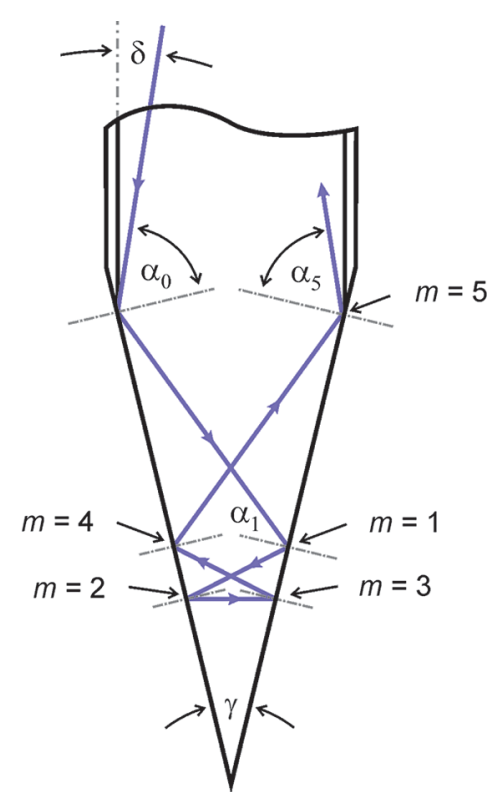

Fig. 7 A schematic presentation of internal reflections in the conical FT. For the sake of clarity, the transmitted beams, defined by the Snell and Fresnel laws, are not drawn. 
of the FT is surrounded by a vapor-layer [see Fig. 5(b)] induced by the absorption of Er:YAG light, the angle of total internal reflection is reduced to $\alpha_{\text {totSV }}=44.7 \mathrm{deg}$. Moreover, according to the Fresnel laws, the portion of reflected light increases due to the greater difference in the refractive indices $n_{c}$ and $n_{m}$.

\subsection{Bubble's Dynamics}

A typical sequence of images of a bubble captured at different times after the beginning of a free-running erbium pulse $\left(E_{L}=40 \mathrm{~mJ}, t_{L}=145 \mu \mathrm{s}\right)$ is presented in Fig. 8. Our experimental results show that a channel-like bubble is formed in the case of the flat FT [Fig. 8(a)], while a spherical bubble develops when a conical FT is used [Fig. 8(b)]. We assume that the shape of the bubble is correlated with the emission profile at the end of the FT. The conical termination of the optical fiber causes a more divergent output profile, ${ }^{14}$ which leads to a spherical bubble.

As we have already explained in the previous subsection, the laser-energy deposition due to the strong absorption of the erbium radiation causes superheated water and its boiling induces a vapor bubble. The expansion, driven by rapid heating, can generate thermo-elastic stress waves. ${ }^{35}$ This happens when the laser energy is deposited into a medium within a time that is shorter than the characteristic time $t_{s}$ for the propagation of stress waves across the irradiated volume: ${ }^{41,43}$

$$
t_{s}=\frac{1}{\mu_{a} c_{s}} \approx 0.5 \mathrm{~ns} .
$$

In the above equation, $c_{s}=1,480 \mathrm{~ms}^{-1}$ stands for the sound velocity in the water. In our case, the vapor is induced in the time interval $t_{v f}<1.5 \mu \mathrm{s}$, which is three orders of magnitude larger than $t_{s}$. Therefore, the stress-confinement conditions are not achieved, so the stress waves are not induced. This is proved by the shadowgraphic observation, where shock waves are not observed during the first bubble expansion [e.g., see the images A1-C1 in Fig. 3(a) and 3(b)]. This finding is also consistent with the pressure measurements performed with a hydrophone presented in the paper by Frenz et al. ${ }^{37}$ However, their laser had a wavelength of $\lambda=2.12 \mu \mathrm{m}$, so the characteristic time $t_{s}=0.2 \mu \mathrm{s}$ in their case is comparable to the time of the vapor formation. For this reason, they observed a slow increase in the pressure during the first bubble expansion.

After the laser-induced boiling, the high pressure of the vapor leads to the bubble's rapid expansion, observed in images B1-C1 in Fig. 8(a) and B1-D1 in Fig. 8(b). When a vapor bubble is formed the laser radiation is transmitted through the expanding bubble and is absorbed at its opposite side. ${ }^{3,36}$ Therefore, the bubble is not only vapor-driven; it is also pulsedriven, since its energy increases due to the absorption of laser energy at the bubble's wall. Here, each individual laser spike contributes to the bubble growth through the evaporation, which happens immediately after the irradiated water reaches the boiling point. ${ }^{22}$

During the expansion, the bubble passes over the equilibrium state. Thus, at its maximum volume [e.g., see images D1 in Fig. 8(a) and A2 in Fig. 8(b)], the internal pressure is lower than the pressure in the surrounding liquid. This difference in pressures forces the bubble to collapse [see images B2-D2 in Fig. 3(a) and B2-C2 in Fig. 8(b)]. During the collapse, a portion of the energy stored in the bubble is converted into acoustic energy. This results in the emission of acoustic transients, shock waves (SW), labeled in images D2 in Fig. 8(a) and C2 in Fig. 8(b). The bubble's oscillations are repeated several times and a new shock wave is emitted after each bubble's rebound [e.g., see images B3 in Fig. 8(a) and 8(b)]. The pressures of the shock waves induced during the bubble's expansion and rebounds were measured by different authors ${ }^{22,32,35,37}$ using a hydrophone. Their results show that in the case of freerunning-mode pulses, the high-pressure transients exist only during rebounds. This is consistent with the shadowgraphic observations in our experiments.

The horizontal arrows in Fig. 8 indicate the FT's end. In case of the flat FT [Fig. 8(a)], the laser radiation ablates a cylindrical channel into the water. As is visible in images $\mathrm{C} 1-\mathrm{A} 2$ in

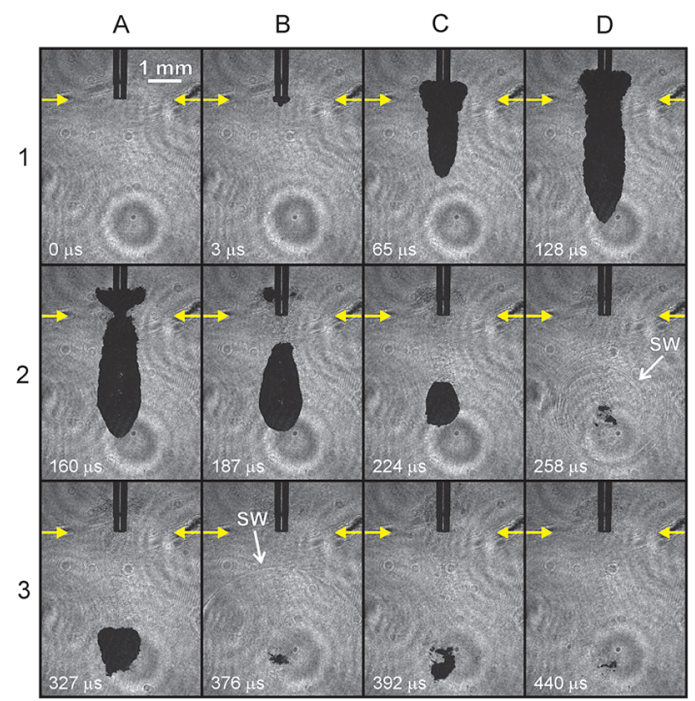

(a)

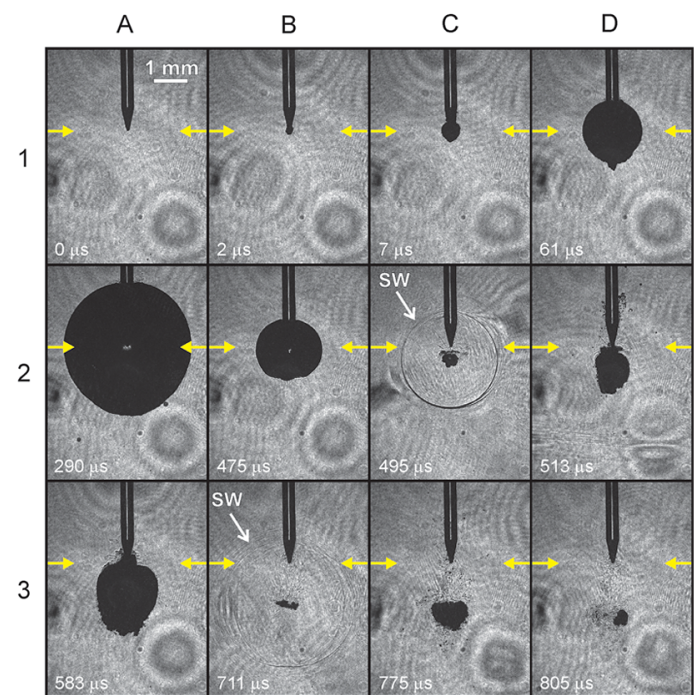

(b)

Fig. 8 Typical images of vapor bubbles induced by erbium radiation $\left(E_{L}=40 \mathrm{~mJ}, t_{L}=145 \mu \mathrm{s}\right)$ delivered through different fiber tips. (a) Flat FT: the bubble has a channel-like shape. (b) Conical FT: a spherical bubble is developed. The scale is shown in the upper-right corner of images A1. The horizontal arrows indicate the FT's end. 
Fig. 8(a), this bubble not only grows in the downward direction due to the pulse vaporization, but also expands up along the fiber. Moreover, the top part of this channel-like bubble is wider compared to the rest of the bubble. The reason for this lies in the higher pressure at the beginning of the bubble's formation.

During the collapse, the bubble is divided into two parts [see image B2 in Fig. 8(a)]. For the laser parameters used in Fig. 8, only the bottom part experiences several rebounds [images B2-C3 in Fig. 8(a)]. In contrast, the top part, which collapses near the FT, already decays during the first collapse.

On the other hand, when the pulse is delivered through the conical FT, we observed a spherical bubble [Fig. 8(b)]. Here, the center of the bubble within the first expansion and the first collapse coincides with the point of the conical FT [images B1-B2 in Fig. 8(b)]. However, the shock wave generated after the first bubble's collapse [see image $\mathrm{C} 2$ in Fig. 8(b)] has its center below the FT's point. This indicates that the bubble's center is moved before the second expansion of the bubble, since the acoustic transient is emitted at the end of the bubble's collapse. ${ }^{44}$

\subsubsection{Volume as a function of time}

Applying the image processing described in the Sec. 2.2, the bubble's volume $V_{B}$ can be obtained from shadowgraphs using Eqs. (1)-(3). A typical bubble's volume as a function of time for two different pulse parameters and both FT geometries is presented in Fig. 9. Here, we show the results for the same pulse energy $E_{L}=20 \mathrm{~mJ}$, but different pulse durations: $t_{L}=120 \mu \mathrm{s}$ (the squares) and $t_{L}=60 \mu \mathrm{s}$ (the circles). The vertical lines mark the end of the excitation pulse. The pulse power as a function of time for the data in Fig. 9 is shown in Fig. 4. The effect of pulse shortening is clearly visible. If the pulse duration is reduced while keeping constant pulse energy, the bubble's maximum volume increases. It can be also concluded from Fig. 9 that the bubbles induced through the conical FT (the full data marks) reach a larger maximum volume in comparison to those formed through the flat FT (the open data marks).

\subsection{Influence of the Pulse Duration on the Bubble's Expansion}

The expansion of a spherical bubble in an infinitely large, nonviscous, and incompressible liquid can be roughly described by the Rayleigh-Plesset equation: ${ }^{45}$

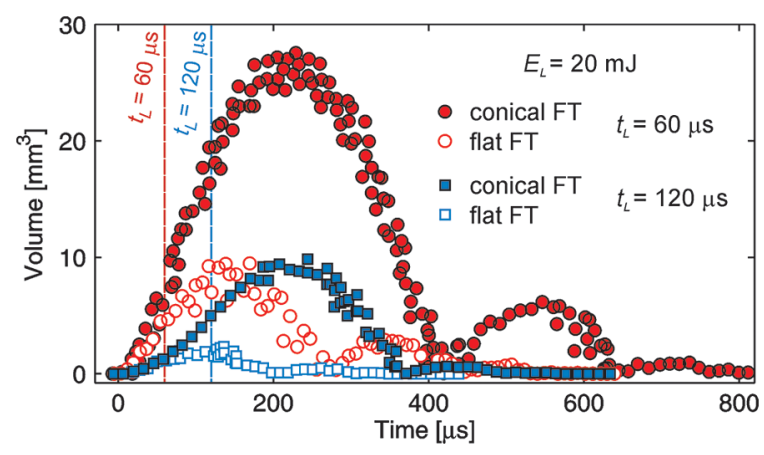

Fig. 9 Bubble's volume as a function of time. Measurements are shown for both FT geometries and two pulse durations $\left(t_{L}=60 \mu \mathrm{s}\right.$ and $\left.t_{L}=120 \mu \mathrm{s}\right)$ at an unchanged pulse energy of $20 \mathrm{~mJ}$ on the exit of the FT.

$$
r \ddot{r}+\frac{3}{2} \dot{r}^{2}=\frac{p_{r}(t)-p_{\infty}(t)}{\rho},
$$

where the dot stands for a time derivative, $r$ is the bubble's radius as a function of time, $\rho$ is the liquid's density, and $p_{r}(t)-$ $p_{\infty}(t)=-\Delta p(t)$ the pressure difference between that at the bubble's wall $p_{r}(t)$, and the pressure of the surrounding liquid $p_{\infty}(t)$. If the pressure difference $\Delta p$ is constant, Eq. (10), which is a general equation of motion for a spherical bubble, simplifies into the well-known Rayleigh equation: ${ }^{33}$

$$
\dot{r}^{2}=\frac{2 \Delta p}{3 \rho}\left(\frac{r_{\max }^{3}}{r^{3}}-1\right) .
$$

Here, $r_{\max }$ stands for the maximum radius of the bubble.

The spherical bubbles are induced through the conical FT. For this reason, we used these measurements to analyze the influence of pulse duration on the expansion of vapor bubbles. In this case, the bubble's radius $r$ as a function of time can be calculated from the measured volume $V_{B}(t)$ as:

$$
r(t)=\left(\frac{3}{4 \pi} V_{B}(t)\right)^{1 / 3} .
$$

For a better interpretation of the experimental results, Eq. (11) should be rewritten in a dimensionless form using the following normalization: $\xi=r / r_{\max }$ and $\tau=t / t_{\max } \cdot{ }^{39}$ Here, $t_{\max }$ stands for the time when the bubble reaches its maximum radius $r_{\max }$.

The integration of Eq. (11) yields the following expression in a dimensionless form:

$$
\begin{aligned}
\tau(\xi) & =\frac{1}{B(5 / 6,1 / 2)} \int_{0}^{\xi^{3}} z^{-1 / 6}(1-z)^{-1 / 2} \mathrm{~d} z \\
& =I_{\xi^{3}}(5 / 6,1 / 2)
\end{aligned}
$$

where $B$ and $I_{\xi^{3}}$ denote the Beta and the incomplete Beta function, ${ }^{46}$ respectively.

When cavitation bubbles in an infinite liquid are induced by short (i.e., nanosecond) laser pulses, their dynamics conforms to the Rayleigh model and can be unified in the dimensionless graph $\xi(\tau) \cdot{ }^{39,47}$ This confirms that the assumption of a constant pressure difference $\Delta p$ during the bubble's expansion is reasonable in the case of short pulses. In contrast, when the pulse duration is comparable to the bubble-expansion time, the evaporation takes place in a liquid shell due to the pulse absorption during the bubble's growth. We analyzed the influence of the pulse duration on the bubble's dynamics by a comparison of the measured results with the predictions of the Rayleigh model [Eq. (13)].

Figure 10 shows the dimensionless radius as a function of the dimensionless time for the spherical bubbles induced by the pulses with durations of $60 \mu \mathrm{s}$ (the crosses), $145 \mu \mathrm{s}$ (the dots), and $285 \mu$ s (the pluses). Here, the measured data are normalized with the maximum measured radii $r_{\max }$ and the measured times $t_{\max }$. The pulse durations $\tau_{L}$ in a dimensionless form are also presented and the solid curve shows the Rayleigh model [Eq. (13)]. It can be concluded that the measured bubble's expansion approaches the Rayleigh model when the pulse duration decreases (i.e., $\tau_{L} \rightarrow 0$ ). In contrast, for longer pulses, the "pulse-driven effect" is clearly visible. In this case, the experimental results do not conform to the Rayleigh model, due to the evaporation induced by the light absorption. Therefore, the 


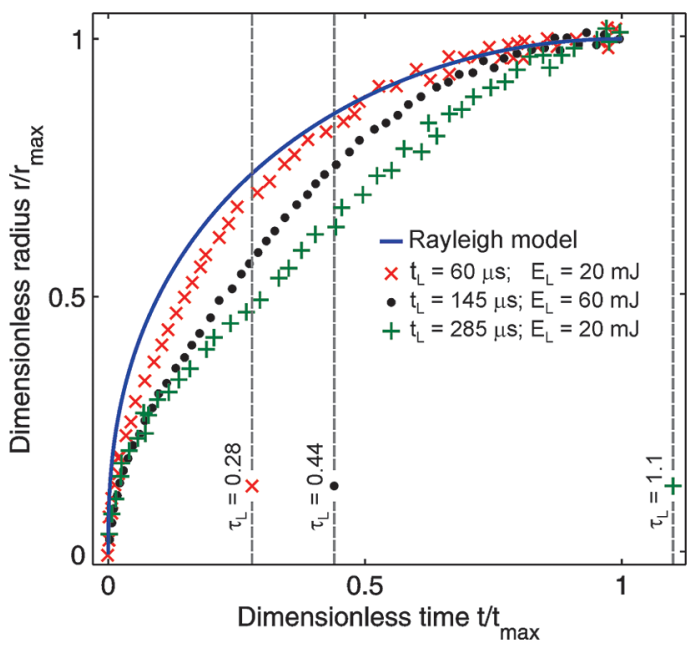

Fig. 10 Dimensionless radii as a function of the dimensionless time for spherical bubbles induced through the conical FT. $\tau_{L}$ labels normalized pulse durations.

assumption of a constant pressure difference $\Delta p$ during the bubble's expansion is no longer valid.

\subsection{Optodynamic Energy-Conversion Efficiency}

In our case, the shock wave during the bubble's formation is not present. This finding is important due to two reasons: (i) it shows that at the wavelength of $2.94 \mu \mathrm{m}$ the shock waves cannot be the main mechanism for cleaning the root canal, as suggested in Refs. 15, 16, 17; and (ii) it means that the total mechanical energy of the liquid medium equals the mechanical energy of the first bubble expansion.

When the bubble reaches its maximum volume, its radial velocity vanishes. The surface tension for millimeter-sized bubbles can be neglected, so a bubble at its maximum radius possesses only a potential energy. ${ }^{48}$ Thus, the total energy of the bubble $E_{B}$ can be obtained as the product of its maximum volume $V_{\max }$ and the difference between the hydrostatic and internal (vapor) pressure. ${ }^{49,50}$ Since the vapor pressure at $V_{\max }$ is much smaller than the hydrostatic pressure, ${ }^{51}$ it can be neglected. Thus, the bubble's energy can be estimated using the following equation: ${ }^{39}$

$$
E_{B}=p_{\infty} V_{\max },
$$

where, $p_{\infty}=10^{5} \mathrm{~Pa}$ equals the hydrostatic pressure of the water. The OD energy-conversion efficiency $\eta$ is therefore defined as:

$$
\eta=\frac{E_{B}}{E_{L}}=\frac{p_{\infty} V_{\max }}{E_{L}} .
$$

We determined $V_{\max }$ from measurements of the volume as a function of time (e.g., see Fig. 9) as follows. First, we fit the quartic function to the measured data corresponding to volumes larger than $V_{\max } / 2$. Then we determined the time $t_{\max }$ as the time related to the maximum of the fitted function. Finally, $V_{\max }$ is calculated as an average of the volumes measured within the time interval $[0.9,1.1] \times t_{\max }$.

Using Eq. (15), we have analyzed how (i) the pulse energy, (ii) the pulse duration, and (iii) the FT geometry influence the OD energy-conversion efficiency. Figure 11 shows the measured efficiency as a function of the pulse duration,

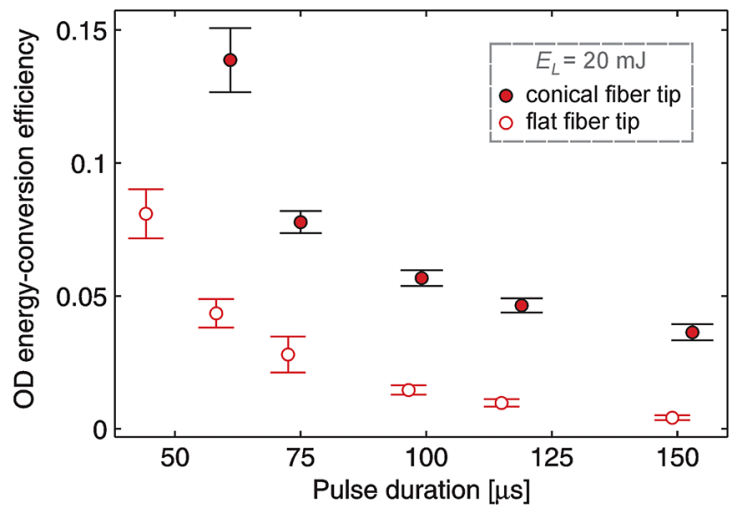

Fig. 11 OD energy-conversion efficiency as a function of pulse duration at a constant pulse energy $\left(E_{L}=20 \mathrm{~mJ}\right)$.

where the pulse energy $\left(E_{L}=20 \mathrm{~mJ}\right)$ was kept constant. On the other hand, Fig. 12 presents the measured efficiency as a function of the pulse energy, where the pulse duration $\left(t_{L}=145 \mu \mathrm{s}\right)$ was constant during the measurements. The results are presented for the conical (the full circles) and the flat (the open circles) FTs. The error bars correspond to the standard deviation calculated from the volume averaging.

It can be concluded from the results presented in Figs. 11 and 12 that the OD energy-conversion efficiency (i) decreases with an increasing pulse duration, (ii) increases by increasing the pulse energy, and (iii) is significantly larger when a conical FT is used rather than a flat FT. These findings have different meanings for various biomedical applications.

The mechanical effects in water should be minimized when a tissue is ablated in a non-contact mode under water (e.g., see Ref. 22). Here, the water serves as a cooler. Therefore, the longer pulses and a flat FT with a channel-like bubble and lower efficiency are more appropriate for this case.

On the other hand, in the medical treatments such as cleaning root canals, the maximization of the mechanical effects is especially important. ${ }^{16}$ Causing the same mechanical effects, a higher efficiency allows less collateral heating, which increases the temperature of the surrounding medium and results in collateral tissue damage and increasing pain. In this case, it is thus more convenient to use conical FTs instead of the flat ones and to keep the duration of the laser pulses as short as possible. However, when higher pulse powers are necessary (i.e., the pulse energy exceeds $100 \mathrm{~mJ}$ within a pulse duration of less

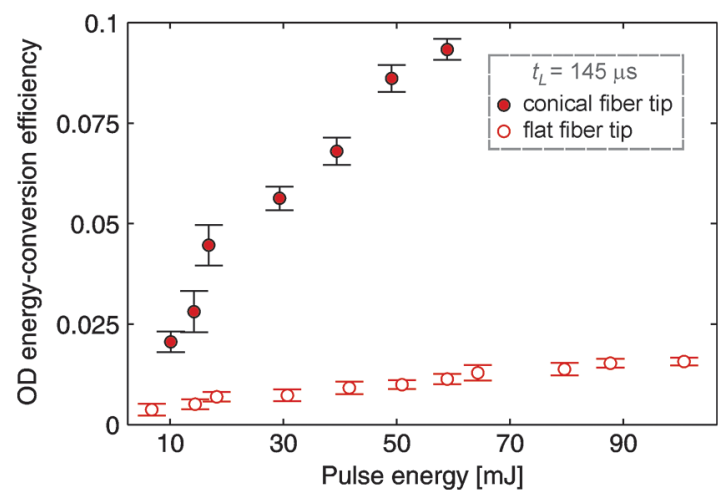

Fig. 12 OD energy-conversion efficiency as a function of pulse energy at a constant pulse duration $\left(t_{L}=145 \mu \mathrm{s}\right)$. 
than $100 \mu \mathrm{s}$ ), the flat FT should be used, because at the point of the conical FT the intensity is increased. Thus, a conical termination experiences damage at lower pulse powers than the flat one.

\section{Conclusions}

We have studied the influence of the FT geometry and the parameters of the free-running erbium pulse on the vapor-bubble formation and dynamics. The experiments were performed using shadow photography. Two different FT terminations, a flat and a conical, were used. The shadowgraphs showed that the bubble's shape depends on FT geometry. A spherical bubble develops when the conical FT is used, while a channel-like bubble is obtained with the flat one. In both cases, bubbles experience several oscillations and a shock wave is emitted during each collapse.

During the first bubble expansion, the shock wave is not present. Therefore, the total mechanical energy of the liquid equals the energy of the first bubble's expansion. This enables us to define the OD energy-conversion efficiency as the ratio between the bubbles' energy and the energy of the laser pulse. Our results show that the efficiency increases (i) by increasing the pulse energy and (ii) decreasing the pulse duration. Moreover, it is larger when a conical FT is used rather than a flat one. The presented results are important for the optimization of biomedical processes, where the collateral heating of the surrounding medium should be reduced.

\section{Acknowledgments}

We would like to thank Fotona, d.d. for supplying their commercially available laser system for laser cleaning root canals in clinical dentistry applications. Their system, including the Er:YAG laser (LightWalker), handpiece, and interchangeable fiber tips was used as an excitation source in our experiments.

\section{References}

1. R. Brinkmann et al., "Analysis of cavitation dynamics during pulsed laser tissue ablation by optical on-line monitoring," IEEE J. Sel. Top. Quant. 2(4), 826-835 (1996).

2. H. Pratisto et al., "Combination of fiber-guided pulsed erbium and holmium laser radiation for tissue ablation under water," Appl. Opt. 35(19), 3328-3337 (1996).

3. T. Lü et al., "Cavitation effect of holmium laser pulse applied to ablation of hard tissue underwater," J. Biomed. Opt. 15(4), 048002 (2010).

4. T. Perhavec and J. Diaci, "A novel double-exposure shadowgraph method for observation of optodynamic shock waves using fiber-optic illumination," Strojniški Vestnik-J. Mech. Eng. 56(7-8), 477-482 (2010).

5. A. Bizjak, K. Nemeš, and J. Možina, "Rotating-mirror Q-switched Er: YAG laser for optodynamic studies,' Strojniški Vestnik-J. Mech. Eng. 57(1), 3-10 (2011).

6. G. M. Hale and M. R. Querry, "Optical-constants of water in 200-nm to 200- $\mu$ m wavelength region," Appl. Opt. 12(3), 555-563 (1973).

7. K. F. Chan et al., "Erbium:YAG laser lithotripsy mechanism," J. Urol. 168(2), 436-441 (2002).

8. H. Petersen, M. Mrochen, and T. Seiler, "Comparison of erbium: yttrium-aluminum-garnet-laser vitrectomy and mechanical vitrectomy," Ophthalmology 107(7), 1389-1392 (2000).

9. M. Mrochen et al., "Erbium:yttrium-aluminum-garnet laser induced vapor bubbles as a function of the quartz fiber tip geometry," J. Biomed. Opt. 6(3), 344-350 (2001).

10. A. Mehl et al., "Bactericidal effects of $2.94 \mu \mathrm{m}$ Er:YAG-laser radiation in dental root canals," J. Endodont. 25(7), 490-493 (1999).

11. H. Tachinami and I. Katsuumi, "Removal of root canal filling materials using Er:YAG laser irradiation," Dent. Mater. J. 29(3), 246-252 (2010).
12. H. Matsumoto, Y. Yoshimine, and A. Akamine, "Visualization of irrigant flow and cavitation induced by Er:YAG laser within a root canal model," J. Endodont. 37(6), 839-843 (2011).

13. R. George and L. J. Walsh, "Performance assessment of novel side firing safe tips for endodontic applications," J. Biomed. Opt. 16(4), 048004 (2011).

14. R. George and L. J. Walsh, "Performance assessment of novel side firing flexible optical fibers for dental applications," Lasers Surg. Med. 41(3), 214-221 (2009).

15. D. L. Glover et al., "Laser based enhanced generation of photoacoustic pressure waves in dental and medical treatments and procedures," U.S. Patent No. 7959441 B2 (2011).

16. E. DiVito, O. A. Peters, and G. Olivi, "Effectiveness of the Erbium:Yag laser and new design radial and stripped tips in removing the smear layer after root canal instrumentation," Laser. Med. Sci. 27(2), 273-280 (2012).

17. G. Olivi and E. DiVito, "Photoacoustic endodontics using PIPS" experimental background and clinical protocol,' J. Laser Health Acad. 2012(1), 22-25 (2012).

18. C. P. Lin, D. Stern, and C. A. Puliafito, "High-speed photography of Er-Yag laser ablation in fluid - implication for laser vitreous surgery," Invest. Ophth. Vis. Sci. 31(12), 2546-2550 (1990).

19. E. D. Jansen et al., "Partial vaporization model for pulsed mid-infrared laser ablation of water,' J. Appl. Phys. 78(1), 564-571 (1995).

20. A. Vogel and V. Venugopalan, "Mechanisms of pulsed laser ablation of biological tissues," Chem. Rev. 103(2), 577-644 (2003).

21. H. Loertscher, W. Q. Shi, and W. S. Grundfest, "Tissue ablation through water with erbium:YAG lasers," IEEE T. Biomed. Eng. 39(1), 86-88 (1992).

22. M. Ith et al., "Dynamics of laser-induced channel formation in water and influence of pulse duration on the ablation of biotissue under water with pulsed erbium-laser radiation," Appl. Phys. B 59(6), 621-629 (1994).

23. E. A. Brujan et al., "Dynamics of laser-induced cavitation bubbles near an elastic boundary," J. Fluid Mech. 433, 251-281 (2001).

24. P. Gregorčič et al., "Measurements of cavitation bubble dynamics based on a beam-deflection probe," Appl. Phys. A 93(4), 901-905 (2008).

25. R. Evans et al., "Pump-probe imaging of nanosecond laser-induced bubbles in agar gel," Opt. Express 16(10), 7481-7492 (2008).

26. A. B. Karpiouk et al., "Quantitative ultrasound method to detect and monitor laser-induced cavitation bubbles," J. Biomed. Opt. 13(3), 034011 (2008).

27. W. Lauterborn and T. Kurz, "Physics of bubble oscillations," Rep. Prog. Phys. 73(10), 106501 (2010).

28. A. Vogel et al., "Intraocular Nd-Yag laser-surgery-light tissue interaction, damage range, and reduction of collateral effects," IEEE Quant. Elect. 26(12), 2240-2260 (1990).

29. F. G. Perez-Gutierrez, S. Camacho-Lopez, and G. Aguilar, "Timeresolved study of the mechanical response of tissue phantoms to nanosecond laser pulses," J. Biomed. Opt. 16(11), 115001 (2011).

30. P. Gregorčič, R. Petkovšek, and J. Možina, "Investigation of a cavitation bubble between a rigid boundary and a free surface," J. Appl. Phys. 102(9), 094904 (2007).

31. T. G. van Leeuwen et al., "Noncontact tissue ablation by holmium: YSGG laser-pulses in blood," Laser. Surg. Med. 11(1), 26-34 (1991).

32. T. Asshauer, K. Rink, and G. Delacretaz, "Acoustic transient generation by holmium-laser-induced cavitation bubbles," J. Appl. Phys. 76(9), 5007-5013 (1994).

33. L. Rayleigh, "On the pressure developed in a liquid during the collapse of a spherical cavity," Philos. Mag. 34(200), 94-98 (1917).

34. E. A. Brujan and A. Vogel, "Stress wave emission and cavitation bubble dynamics by nanosecond optical breakdown in a tissue phantom," J. Fluid Mech. 558, 281-308 (2006).

35. E. D. Jansen et al., "Effect of pulse duration on bubble formation and laser-induced pressure waves during holmium laser ablation," Laser. Surg. Med. 18(3), 278-293 (1996).

36. T. Asshauer et al., "Pulsed holmium laser ablation of tissue phantoms: correlation between bubble formation and acoustic transients," Appl. Phys. B 65(4-5), 647-657 (1997).

37. M. Frenz et al., "Starting mechanisms and dynamics of bubble formation induced by a Ho:Yttrium aluminum garnet laser in water," J. Appl. Phys. 84(11), 5905-5912 (1998). 
38. R. Petkovšek, P. Gregorčič, and J. Možina, "A beam-deflection probe as a method for optodynamic measurements of cavitation bubble oscillations," Meas. Sci. Technol. 18(9), 2972-2978 (2007).

39. R. Petkovšek and P. Gregorčič, "A laser probe measurement of cavitation bubble dynamics improved by shock wave detection and compared to shadow photography," J. Appl. Phys. 102(4), 044909 (2007).

40. G. S. Settles, Schlieren and Shadowgraph Techniques, Springer-Verlag, Berlin (2001).

41. S. L. Jacques, "Role of tissue optics and pulse duration on tissue effects during high-power laser irradiation," Appl. Opt. 32(13), 2447-2454 (1993).

42. O. Fohn et al., "Side-firing fiber device for underwater tissue ablation with Ho:YAG and Er:YAG laser radiation," J. Biomed. Opt. 3(1), 112-122 (1998).

43. R. O. Esenaliev et al., "Studies of acoustical and shock-waves in the pulsed-laser ablation of biotissue," Laser. Surg. Med. 13(4), 470-484 (1993).

44. I. Akhatov et al., "Collapse and rebound of a laser-induced cavitation bubble," Phys. Fluids 13(10), 2805-2819 (2001).
45. M. S. Plesset, "The dynamics of cavitation bubbles," J. Appl. Mech. 16, 228-231 (1949).

46. M. Abramowitz and I. A. Stegun, Handbook of Mathematical Functions, Dover Publications, New York (1972).

47. S. W. Gong et al., "Scaling law for bubbles induced by different external sources: Theoretical and experimental study," Phys. Rev. E 81(5), 056317 (2010).

48. W. Kreider et al., "Observations of the collapses and rebounds of millimeter-sized lithotripsy bubbles," J. Acoust. Soc. Am. 130(5), 3531-3540 (2011).

49. A. Vogel, W. Lauterborn, and R. Timm, "Optical and acoustic investigations of the dynamics of laser-produced cavitation bubbles near a solid boundary," J. Fluid Mech. 206, 299-338 (1989).

50. S. Buogo and G. B. Cannelli, "Implosion of an underwater sparkgenerated bubble and acoustic energy evaluation using the Rayleigh model," J. Acoust. Soc. Am. 111(6), 2594-2600 (2002).

51. A. Vogel et al., "Energy balance of optical breakdown in water at nanosecond to femtosecond time scales," Appl. Phys. B 68(2), 271-280 (1999). 